
25 Research Square
Preprints are preliminary reports that have not undergone peer review.
They should not be considered conclusive, used to inform clinical practice, or referenced by the media as validated information.

\title{
Sirtuin3 rs28365927 functional mutation confers to the high risk of non- alcoholic fatty liver disease in Chinese Han population
}

\author{
Li-Jie Chen \\ Central South University \\ Jing Guo \\ Central South University \\ Song-xia Zhang \\ Central South University \\ Ying $\mathrm{Xu}$ \\ Central South University \\ Qing Zhao \\ Central South University \\ Wei Zhang \\ Central South University \\ Jian Xiao \\ Central South University \\ Yao Chen ( $\nabla$ cbohua@csu.edu.cn) \\ Central South University
}

\section{Research Article}

Keywords: Sirtuin3, non-alcoholic fatty liver disease, functional mutation, rs28365927

Posted Date: July 8th, 2021

DOI: https://doi.org/10.21203/rs.3.rs-680336/v1

License: @ (i) This work is licensed under a Creative Commons Attribution 4.0 International License. Read Full License 


\section{Abstract \\ Background}

Non-alcoholic fatty liver disease (NAFLD) is a multifactorial condition associated with aging, insulin resistance, metabolic syndrome, genetic factors and more. Although genetic traits are among the most important risks factors for NAFLD, the understanding of their influence is still quite limited. The present study aimed at identifying novel SNPs that may confer a risk for NAFLD in the Han Chinese population.

\section{Methods}

Based on the "two-hit hypothesis", candidate SNPs, including Sirtuin3 rs28365927, were genotyped by MassARRAY in B-type ultrasonography-proven NAFLD patients $(n=292)$ and healthy controls $(n=387)$.

\section{Results}

In a model analysis of individuals matched based on gender and age that compared 223 NAFLD and 223 non-NAFLD patients, the rs28365927 GA + AA genotype was a significant risk factor for the development of NAFLD in a dominant model. Rs28365927 was significantly associated with a higher NAFLD risk in both an additive model (A vs G) and genotypic model (GA vs GG). Among the NAFLD patients, serum levels of total bilirubin (TBIL), DBIL direct bilirubin (DBIL) and glutamic-pyruvic transaminase (ALT) in rs28365927 A allele carriers (GA + AA) were $11.1 \%, 14.7 \%$ and $41.5 \%$ higher, respectively, than in noncarriers (GG). Furthermore, among the NAFLD patients, the carriers of Rs28365927 allele A were positively correlated with higher ALT levels.

\section{Conclusion}

Sirtuin3 rs28365927 is an independent risk factor for NAFLD in the Han Chinese population.

\section{Introduction}

Non-alcoholic fatty liver disease (NAFLD) is a common cause of liver-related death that affects a quarter of adults worldwide, with the highest prevalence in the Middle East (31.8\%) and South America (30.5\%) and the lowest in Africa (13.5\%). Furthermore, the prevalence of NAFLD in China is 27.4\%[1]. This disease is characterized by an excess accumulation of fat (in the form of triglycerides) in the hepatocytes (> $\%$ fat content in the liver, referred to as steatosis), leading to non-alcoholic fatty liver[2]. NAFLD includes a broad range of pathologic features, ranging from benign and reversible simple steatosis to more severe nonalcoholic steatohepatitis (NASH), which can progress to fibrosis, cirrhosis and even hepatocellular carcinoma[3]. NAFLD-related diabetes, atherosclerosis and liver disease have significantly increased complications and mortality, posing a serious threat to life and health[4].

NAFLD is a multifactorial disease with obvious individual susceptibility, and a number of risk factors have been identified, such as aging, insulin resistance, hyperlipidaemia, obesity, type 2 diabetes, dyslipidaemia, genetic factors and other conditions[5-11]. Genetic factors are among the most important determinants for individual susceptibility to NAFLD, and great progress has been made in identifying these factors in recent years. For example, rs738409, a functional loss variant of patatin-like phospholipase domain containing 3 (PNPLA3) (I148M), has been associated with the severity of steatosis and fibrosis as well as the presence of NASH [12]. The GCKR rs780094 (P446L) mutation increases the accumulation of liver fat by stimulating fat generation and glucose uptake[13]. In the Han Chinese population, SREBP-2 rs2228314 carriers of G (CG + GG) may be at an increased risk for NAFLD[14].

Although considerable progress has been made in the study of individual susceptibilities to NAFLD, much is still unknown. Therefore, the discovery of new single nucleotide polymorphisms (SNPs) associated with NAFLD risk still remains of great significance for the prediction and prevention of individual susceptibility to this disease[15]. The purpose of the current study was to discover new SNPs that contribute to the individual susceptibility risk for NAFLD in the Han Chinese population.

\section{Subjects And Methods}

\subsection{Study subjects}

This case-control retrospective study was performed in accordance with the principles of the Declaration of Helsinki and its appendices. It was approved by the Medical Ethics Committee of the Department of Clinical Pharmacology, Xiangya Hospital, Central South University (Changsha, China) and was registered with the Chinese Clinical Trial Registry (no. ChiCTR-ROC-15006899). All subjects gave written and informed consent before participating in this study.

From June 2015 to January 2020, 292 NAFLD patients and 387 non-NAFLD patients were enrolled in this study. Participants were collected from the Health Management Center of Xiangya Hospital. All of the individuals were unrelated and of Han Chinese origin. The diagnosis of NAFLD was performed according to the guidelines for the diagnosis and treatment of NAFLD of China (2010), the European Association for the Study of the Liver and the American Association for the Study of Liver Diseases criteria[16]. The inclusion criteria were as follows: B-type ultrasonography of the liver showing normal sonography or hepatic imaging findings consistent with the diagnostic criteria for diffuse fatty liver without other explanations and serum glutamic-pyruvic transaminase (ALT) and/or glutamic oxaloacetic transaminase (AST) and gamma-glutamyltransferase (GGT) levels continuously raised for over half a year in patients with components related to metabolic syndrome with unknown causes. All subjects with other causes of liver disease were excluded, including excess alcohol 
intake ( $\geq 20 \mathrm{~g} / \mathrm{d})$, viral liver infection, hepatic cyst, liver haemangioma, drug-induced hepatitis, schistosomal liver, liver calcifications, cirrhosis and other liver diseases.

Venous blood samples (3-5 mL/each) were collected from each patient for DNA extraction using disposable venous blood lancets and disposable blood collection tubes. Blood genomic DNA was isolated with a commercial DNA extraction kit (Omega Bio-Tek, GA, USA) according to the manufacturer's instructions and stored at $-80^{\circ} \mathrm{C}$ until use. The participants' clinical and demographic data, including age, gender, body mass index (BMI), systolic blood pressure (SBP), diastolic blood pressure (DBP), waist circumference (WC), hip circumference (HC) and biochemical laboratory parameters, were also recorded (Fig. 1).

\subsection{Genetic variants selection and genotyping}

The genes affecting individual susceptibility risk for NAFLD were selected based on the "two-hit theory". Among these, seven genes (MTTP, PEMT, FASN, PGC1 $\beta$, ApoE, mTOR and SLC27A5) were associated with the "first hit", and 10 genes (PNPLA3, Sirtuin3, CYP2E1, Fas, TLR4, TCF7L2, PPARG, IL6, STAT2 and HIF3A) were associated with the "second hit" (Fig. 2). Further screening was performed for SNPs affecting the functioning of these genes based primarily on the functional mutations identified in the ENCODE database (http://genome.ucsc.edu/ENCODE/) while also meeting a Han Chinese population minor allele frequency of $>0.05$ in the 1000 Genomes database, resulting in the selection of a total of 17 candidate functional SNPs to be sent to MassARRAY for sequencing (Table 1). 
Table 1

Allele and genotype distribution of 17 SNPs in NAFLD patients and Non-NALFD patients.

\begin{tabular}{|c|c|c|c|c|c|c|c|c|c|}
\hline $\begin{array}{l}\text { Gene } \\
\text { name }\end{array}$ & SNP & $\begin{array}{l}\text { Nucleotide } \\
\text { change }\end{array}$ & $\begin{array}{l}\text { Protein } \\
\text { change }\end{array}$ & $\begin{array}{l}\text { Variant } \\
\text { allele }\end{array}$ & Wild-type allele & $\begin{array}{l}\mathrm{p}- \\
\text { value }\end{array}$ & $\begin{array}{l}\text { Homozygote } \\
\text { genotype }\end{array}$ & $\begin{array}{l}\text { Heterozygote } \\
\text { genotype }\end{array}$ & $\begin{array}{l}\text { Wild-type } \\
\text { genotype }\end{array}$ \\
\hline & & & & NAFLD/Non & NAFLD/Non & & NAFLD/Non & NAFLD/Non & NAFLD/Non \\
\hline & & & & -NAFLD(\%) & -NAFLD(\%) & & -NAFLD(\%) & -NAFLD(\%) & -NAFLD(\%) \\
\hline PPARG & rs1801282 & $\underset{>G}{g .12393125 C}$ & Pro12Ala & $4.0 \% / 3.5 \%$ & $96.0 \% / 96.5 \%$ & 0.659 & $-/-$ & $8.0 \% / 7.1 \%$ & $92.0 \% / 92.9 \%$ \\
\hline STAT2 & rs2066811 & $\begin{array}{l}\text { g. } 56742997 T \\
>C\end{array}$ & p.lle464Val & $-/-$ & $100.0 \% / 100.0 \%$ & - & - & $-/$ & $100.0 \% / 100.0$ \\
\hline IL6 & rs2069849 & $\begin{array}{l}\text { g. } 22771156 \mathrm{C} \\
>\mathrm{T}\end{array}$ & p.Phe201Leu & $0.4 \% / 0.1 \%$ & $99.6 \% / 99.9 \%$ & 0.401 & $-/-$ & $0.7 \% / 0.3 \%$ & $99.3 \% / 99.7 \%$ \\
\hline FASN & rs2228305 & $\begin{array}{l}\text { g. } 80042792 \mathrm{C} \\
>\mathrm{T}\end{array}$ & p.Val1483lle & $0.9 \% / 0.3 \%$ & $99.1 \% / 99.7 \%$ & 0.124 & $0.7 \% /-$ & $0.4 \% / 0.5 \%$ & $98.9 \% / 99.5 \%$ \\
\hline MTTP & rs2306985 & $\begin{array}{l}\text { g. } 100516022 C \\
>G\end{array}$ & p.His297GIn & $67.1 \% / 65.3 \%$ & $32.9 \% / 34.7 \%$ & 0.477 & $44.8 \% / 43.3 \%$ & $44.8 \% / 43.9 \%$ & $10.5 \% / 12.8 \%$ \\
\hline SIRT3 & rs28365927 & g. $236091 G>A$ & p.Arg80Trp & $14.3 \% / 11.4 \%$ & $85.7 \% / 88.6 \%$ & 0.127 & $1.1 \% / 2.1 \%$ & $26.4 \% / 18.7 \%$ & $72.5 \% / 79.2 \%$ \\
\hline mTOR & rs28990992 & $\begin{array}{l}\text { g.11249789G } \\
>C\end{array}$ & p.Glu51Asp & $4.4 \% \varangle 4.4 \%$ & $95.6 \% / 95.6 \%$ & 0.963 & $0.7 \% / 0.8 \%$ & $7.4 \% / 7.3 \%$ & $91.9 \% / 91.9 \%$ \\
\hline Fas & rs3218619 & $\begin{array}{l}\text { g. } 90762801 G \\
>A\end{array}$ & p.Ala16Thr & $-/-$ & $100.0 \% / 100.0 \%$ & - & $-/-$ & $-/-$ & $100.0 \% / 100.0$ \\
\hline SLC27A5 & rs35350976 & $\begin{array}{l}\text { g. } 59023174 A \\
>G\end{array}$ & p.Met50Thr & $5.6 \% / 4.5 \%$ & $94.4 \% / 95.5 \%$ & 0.381 & $0.4 \% / 0.3 \%$ & $10.5 \% / 8.6 \%$ & $89.1 \% / 91.2 \%$ \\
\hline ApoE & rs440446 & $\begin{array}{l}\text { g. } 45409167 \mathrm{C} \\
>\mathrm{G}\end{array}$ & p.Asn14Lys & $42.0 \% / 39.1 \%$ & $58.0 \% / 60.9 \%$ & 0.279 & $17.7 \% / 14.9 \%$ & $48.6 \% / 48.4 \%$ & $33.7 \% / 36.8 \%$ \\
\hline TLR4 & rs4986790 & $\begin{array}{l}\text { g. } 120475302 A \\
>G\end{array}$ & p.Asp299Gly & $-/ 0.1 \%$ & $100.0 \% / 99.9 \%$ & 0.747 & $-/-$ & $-/ 0.3 \%$ & $100.0 \% / 99.7 \%$ \\
\hline CYP2E1 & rs6413419 & $\begin{array}{l}\text { g. } 135345675 G \\
>T T\end{array}$ & p.Val179Phe & $-/-$ & $100.0 \% / 100.0 \%$ & - & $-/-$ & $-/-$ & $100.0 \% / 100.0$ \\
\hline PNPLA3 & rs738409 & $\begin{array}{l}\text { g. } 44324727 \mathrm{C} \\
>\mathrm{G}\end{array}$ & p.lle148Met & $38.5 \% / 37.9 \%$ & $61.5 \% / 62.1 \%$ & 0.827 & $18.4 \% / 13.9 \%$ & $40.3 \% / 48.0 \%$ & $41.3 \% / 38.1 \%$ \\
\hline PGC1 $\beta$ & rs7732671 & $\begin{array}{l}\text { g. } 149212243 G \\
>C\end{array}$ & p.Ala203Pro & $5.9 \% / 5.0 \%$ & $94.1 \% / 95.0 \%$ & 0.439 & $-/ 0.5 \%$ & $11.8 \% / 8.9 \%$ & $88.2 \% / 90.6 \%$ \\
\hline TCF7L2 & rs77961654 & $\begin{array}{l}\text { g. } 114925369 C \\
>A\end{array}$ & p.Pro200Thr & $26.4 \% / 25.1 \%$ & $73.6 \% / 74.9 \%$ & 0.594 & $8.6 \% / 7.6 \%$ & $35.7 \% / 35.0 \%$ & $55.7 \% / 57.4 \%$ \\
\hline PEMT & rs7946 & $\begin{array}{l}\text { g. } 17409560 \mathrm{C} \\
>\mathrm{T}\end{array}$ & p.Val212Met & $17.4 \% / 17.6 \%$ & $82.6 \% / 82.4 \%$ & 0.918 & $3.5 \% / 3.2 \%$ & $27.7 \% / 28.8 \%$ & $68.8 \% / 68.0 \%$ \\
\hline HIF3A & rs3764609 & $\begin{array}{l}\text { g. } 46823702 A \\
>G\end{array}$ & p.GIn274Arg & $40.0 \% / 37.2 \%$ & $60.0 \% / 62.8 \%$ & 0.287 & $16.8 \% / 13.4 \%$ & $46.5 \% / 47.6 \%$ & $36.7 \% / 39.0 \%$ \\
\hline
\end{tabular}

Abbreviations: PGC1 $\beta$, peroxisome proliferator-activated receptor-y coactivator-1 $\beta$; FASN, fatty acid synthase; mTOR, mechanistic target of rapamycin; PEMT, phosphatidylethanolamine N-methyltransferase; MTTP, microsomal triglyceride transfer protein; SLC27A5, soluble carrier family 27 member A5; APOE, Apolip cytochrome P450 2E1; PPARG, Peroxisome proliferator-activated receptor-y; TCF7L2, transcription factor 7-like 2; HIF3A, Hypoxia Inducible Factor 3 Alpha Sul phospholipase domain containing 3; FAS, fas cell surface death receptor; SIRT3, Sirtuin3; IL-6, interleukin-6; STAT2, signal transducer and activator of transcri receptor 4;

P-value $\mathbb{0} 0.05$ considered as statistically significant (in bold).

\subsection{Statistical analysis}

The odds ratios (ORs) for the candidate SNPs were first analysed by comparing 292 NAFLD patients (177 males and 114 females, mean age $48.89 \pm 11.57$ years) and 387 non-NAFLD patients (134 males and 251 females, mean age $41.97 \pm 12.17$ years). Since significant differences in gender and age were found between the two groups, an individual matching based on gender and age was performed for an OR evaluation between 223 NAFLD patients (122 males and 101 females, mean age $47.97 \pm 11.01$ years) and 223 non-NAFLD patients (122 males and 101 females, mean age $47.37 \pm 11.41$ years). Statistical analysis was performed using SPSS, version 24.0 (IBM, Chicago, IL, USA). SHEsis for Windows was used to analyse the Hardy-Weinberg equilibrium (HWE) and genotype and allele distributions between the patients and controls[17]. The baseline characteristics of the subjects were compared between the groups using a Student's t-test if the data were normally distributed and otherwise with a Mann-Whitney U test and reported as mean \pm standard deviation. Multiple linear regression analyses were used to assess the interaction between the SNPs and clinical characteristics. The association between the SNPs and NAFLD risk was estimated by computing ORs and $95 \%$ confidence intervals (Cls) from the multivariate logistic regression analyses. $\mathrm{P}<0.05$ was considered as statistically significant. 


\section{Results}

\subsection{Sirtuin3 rs28365927 together with gender and age were associated with a risk for NAFLD in the non-matched model}

The clinical characteristics of the NAFLD and non-NAFLD patients are shown in Table 2. The NAFLD patients had a higher age, ratio of males to females, BMI, SBP, DBP, WC, HC values and serum levels of ALT, AST, fasting blood-glucose (FBG), low density lipoprotein cholesterin (LDL-C), triglyceride (TG) and total cholesterol $(T C)$ than the non-NAFLD patients (all $\mathrm{P}<0.05)$. In addition, serum levels of high density lipoprotein cholesterol (HDL-C), total protein (TP) and albumin in the NAFLD patients were significantly lower than in the non-NAFLD patients $(P<0.05)$. No significant differences in the other parameters were observed between the two groups of patients (all $\mathrm{P}>0.05$ ).

Table 2

Clinical Characteristics of Patients with NAFLD and Non-NAFLD in study population ${ }^{\mathrm{a}}$.

\begin{tabular}{|c|c|c|c|}
\hline Characteristics & $\begin{array}{l}\text { Non-NAFLD patients } \\
(n=387)\end{array}$ & $\begin{array}{l}\text { NAFLD patients } \\
(n=292)\end{array}$ & P Value \\
\hline Age, y & $41.97 \pm 12.17$ & $48.89 \pm 11.57$ & 0.000 \\
\hline Gender,Male /Female & $134 / 251$ & $177 / 114$ & 0.000 \\
\hline ALT, U/L & $19.37 \pm 14.17$ & $33.24 \pm 29.92$ & 0.000 \\
\hline AST, U/L & $22.59 \pm 9.59$ & $29.24 \pm 14.47$ & 0.000 \\
\hline $\mathrm{FBG}, \mathrm{mmol} / \mathrm{L}$ & $5.11 \pm 1.42$ & $5.55 \pm 1.63$ & 0.000 \\
\hline LDL-C, mmol/L & $2.81 \pm 0.80$ & $3.12 \pm 0.83$ & 0.000 \\
\hline $\mathrm{TG}, \mathrm{mmol} / \mathrm{L}$ & $1.34 \pm 0.92$ & $2.51 \pm 1.84$ & 0.000 \\
\hline $\mathrm{TC}, \mathrm{mmol} / \mathrm{L}$ & $4.88 \pm 0.97$ & $5.14 \pm 1.07$ & 0.001 \\
\hline $\mathrm{HDL}-\mathrm{C}, \mathrm{mmol} / \mathrm{L}$ & $1.58 \pm 0.38$ & $1.27 \pm 0.33$ & 0.000 \\
\hline $\mathrm{BMI}, \mathrm{kg} / \mathrm{m}^{2}$ & $22.13 \pm 2.56$ & $26.25 \pm 2.55$ & 0.000 \\
\hline SBP, mmHg & $116.01 \pm 17.21$ & $123.95 \pm 19.82$ & 0.000 \\
\hline DBP, mmHg & $73.43 \pm 11.87$ & $84.07 \pm 15.61$ & 0.000 \\
\hline $\mathrm{WC}, \mathrm{cm}$ & $73.65 \pm 8.12$ & $85.40 \pm 8.29$ & 0.000 \\
\hline $\mathrm{HC}, \mathrm{cm}$ & $89.62 \pm 6.00$ & $95.28 \pm 6.68$ & 0.000 \\
\hline $\mathrm{TP}, \mathrm{g} / \mathrm{L}$ & $73.75 \pm 3.84$ & $72.51 \pm 4.93$ & 0.000 \\
\hline Albumin,g/L & $45.97 \pm 3.09$ & $45.03 \pm 4.54$ & 0.003 \\
\hline Globulin,g/L & $27.73 \pm 3.38$ & $27.88 \pm 5.76$ & 0.667 \\
\hline$A / G$ & $1.69 \pm 0.24$ & $1.67 \pm 0.33$ & 0.444 \\
\hline TBIL, umol/L & $12.87 \pm 8.14$ & $12.80 \pm 6.35$ & 0.915 \\
\hline DBIL, umol/L & $5.22 \pm 2.30$ & $5.47 \pm 3.47$ & 0.260 \\
\hline TBA,umol/L & $3.55 \pm 7.09$ & $4.49 \pm 4.98$ & 0.232 \\
\hline \multicolumn{4}{|c|}{$\begin{array}{l}\text { Abbreviations :BMI Body Mass Index,SBP systemic blood pressure,DBP diastolic blood pressure,WC Waist circumference,HP Hip circumference,TP Total } \\
\text { Protein, A/G the ratio of albumin to globulin, TBIL total bilirubin, DBIL direct bilirubin,TBA total bile acid,ALT glutamic-pyruvic transaminase,AST glutamic } \\
\text { oxalacetic transaminase, FBG fasting blood-glucose, LDL-C low density lipoprotein cholesterin, TG triglyceride, TC total cholesterol, HDL-C high density } \\
\text { lipoprotein cholesterol,NAFLD nonalcoholic fatty liver disease. }\end{array}$} \\
\hline
\end{tabular}

The allele and genotype distributions of the 17 SNPs in the NAFLD and non-NAFLD groups are shown in Table 1 and demonstrate that only the genotype distributions of Sirtuin3 rs28365927 showed significant differences between the two groups $(P<0.05)$. The genotype distributions of Sirtuin3 rs28365927 were in accordance with the HWE in both the NAFLD and non-NAFLD patients (all P >0.05) (Table 1).

Based on an analysis using the four genetic models, which includes dominant, recessive, additive and genotypic models, the correlations between NAFLD susceptibility and the genotype distributions of Sirtuin3 rs28365927 were explored further (Table 4). In the unmatched population, the Sirtuin3 rs28365927 GA + AA genotype was a significant risk factor for the development of NAFLD (OR $=1.976,95 \% \mathrm{Cl}$ : $1.088-3.588, \mathrm{P}=0.025$, after adjusting for age, gender and $\mathrm{BMI})$ in the dominant model. There were no significant correlations between Sirtuin3 rs28365927 and NAFLD risk in the recessive and additive models, but there was a significant association between Sirtuin3 rs28365927 and higher NAFLD risk in the genotypic model $(O \mathrm{R}=1.600,95 \% \mathrm{Cl}$ : $1.061-2.415, \mathrm{P}=0.025$, after adjusting for age and gender, GA vs GG). 
Table 4

Association of rs28365927 with NAFLD based on four genetic models in the Study population ${ }^{a}$.

\begin{tabular}{|c|c|c|c|c|c|c|}
\hline & \multicolumn{2}{|l|}{ Unadjusted } & \multicolumn{2}{|l|}{ Adjusted $^{a}$} & \multicolumn{2}{|l|}{ Adjusted $^{b}$} \\
\hline & OR $(95 \% \mathrm{Cl})$ & P Value & OR(95\% Cl) & P Value & $\mathrm{OR}(95 \% \mathrm{Cl})$ & P Value \\
\hline \multicolumn{7}{|l|}{ unmatched } \\
\hline$G A+A A$ vs $G G$ & $1.489(1.034-2.145)$ & 0.032 & $1.532(1.026-2.287)$ & 0.037 & $1.976(1.088-3.588)$ & 0.025 \\
\hline$A A$ vs $G A+G G$ & $0.491(0.129-1.868)$ & 0.297 & $0.486(0.109-2.163)$ & 0.343 & $1.077(0.123-9.433)$ & 0.947 \\
\hline$A$ vs $G$ & $1.286(0.925-1.786)$ & 0.135 & $1.327(0.925-1.905)$ & 0.124 & $1.555(0.909-2.661)$ & 0.107 \\
\hline GG & 1 & 0.042 & 1 & 0.052 & 1 & 0.204 \\
\hline GA & $1.552(1.066-2.260)$ & 0.022 & $1.600(1.061-2.415)$ & 0.025 & $1.744(0.946-3.215)$ & 0.075 \\
\hline$A A$ & $0.542(0.142-2.068)$ & 0.370 & $0.541(0.121-2.417)$ & 0.421 & $1.225(0.138-10.897)$ & 0.855 \\
\hline \multicolumn{7}{|l|}{ matched } \\
\hline$G A+A A$ vs $G G$ & $1.662(1.054-2.619)$ & 0.029 & $1.655(1.048-2.612)$ & 0.031 & $1.838(1.011-3.341)$ & 0.046 \\
\hline$A A$ vs $G A+G G$ & $1.005(0.201-5.034)$ & 0.995 & $1.004(0.199-5.053)$ & 0.996 & $1.820(0.241-13.761)$ & 0.562 \\
\hline$A$ vs $G$ & $1.523(1.007-2.304)$ & 0.046 & $1.516(1.001-2.295)$ & 0.049 & $1.716(1.001-2.942)$ & 0.050 \\
\hline GG & 1 & 0.082 & 1 & 0.087 & 1 & 0.135 \\
\hline GA & $1.705(1.069-2.721)$ & 0.025 & $1.697(1.062-2.712)$ & 0.027 & $1.822(0.987-3.363)$ & 0.055 \\
\hline AA & $1.127(0.224-5.662)$ & 0.885 & $1.126(0.223-5.687)$ & 0.885 & $2.084(0.273-15.935)$ & 0.479 \\
\hline \multicolumn{7}{|c|}{$\begin{array}{l}\text { Abbreviations:OR, odds ratio; } \text { p-value } ₫ 0.05 \text { considered as statistically significant (in bold);four logistic regression models (dominant:heterozygotes and } \\
\text { variant homozygotes vs. wild homozygotes; recessive:variant homozygotes vs. wild homozygotes and heterozygotes; additive: variant allele vs. wild allel } \\
\text { genotypic: heterozygotes or variant homozygotes vs. wild homozygotes) were used to analyze the SNPs. }\end{array}$} \\
\hline \multicolumn{7}{|c|}{ 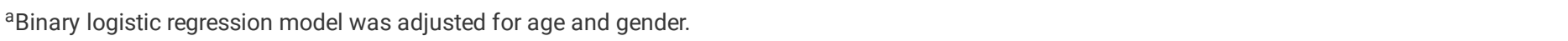 } \\
\hline
\end{tabular}

The above results indicate that age, gender and Sirtuin3 rs28365927 were all associated with NAFLD risk.

\subsection{Sirtuin3 rs28365927 is an independent risk factor for NAFLD in matched populations}

Next, we further verified the association between Sirtuin3 rs28365927 and the risk for NAFLD with age and sex matched between the two groups. There were significant differences in the allele distributions of Sirtuin3 rs28365927 between the NAFLD and non-NAFLD patients ( $P=0.045)$, while the genotype distribution was not significantly different $(P=0.079)$ in the matched populations (Table 3). As shown in Table 4, in the matched populations, the Sirtuin3 rs28365927 GA + AA genotype was a significant risk factor for the development of NAFLD (OR $=1.662,95 \% \mathrm{Cl}: 1.054-2.619, \mathrm{P}=0.029)$. After adjusting for age, gender and $\mathrm{BMI}$, the risk of the Sirtuin3 rs28365927 GA + AA genotype was still marked ( $\mathrm{OR}=1.838,95 \% \mathrm{Cl}: 1.011-3.341, \mathrm{P}=0.046)$ in the dominant model. There was no significant correlation between Sirtuin3 rs28365927 and NAFLD risk in the recessive model, but a significant association was found between Sirtuin3 rs28365927 and higher NAFLD risk in both the additive (OR $=1.716,95 \% \mathrm{Cl}: 1.001-2.942, \mathrm{P}=0.050$, after adjusting for age, gender and $\mathrm{BMI})$ and genotypic models ( $\mathrm{OR}=1.697,95 \% \mathrm{Cl}: 1.062-2.712, \mathrm{P}=0.027$, after adjusting for age and gender, $\mathrm{GA}$ vs $\mathrm{GG})$.

Table 3

Allele and genotype distribution of SIRT3 rs28365927 in NAFLD patients and Non-NALFD patients.

\begin{tabular}{|c|c|c|c|c|c|c|c|c|c|}
\hline & $\begin{array}{l}\text { Variant } \\
\text { Allele(A) }\end{array}$ & $\begin{array}{l}\text { Wild-type } \\
\text { allele(G) }\end{array}$ & $\begin{array}{l}\mathrm{p}- \\
\text { value }\end{array}$ & $\begin{array}{l}\text { Homozygote } \\
\text { genotype(AA) }\end{array}$ & $\begin{array}{l}\text { Heterozygote } \\
\text { genotype(AG) }\end{array}$ & $\begin{array}{l}\text { Wild-type } \\
\text { genotype(GG) }\end{array}$ & $\begin{array}{l}\text { p-value for HWE test } \\
\text { (NAFLD/Non-NAFLD) }\end{array}$ & $\begin{array}{l}\text { p- } \\
\text { value }\end{array}$ & $\begin{array}{l}\text { Call } \\
\text { rate(\%) }\end{array}$ \\
\hline & NAFLD/Non & NAFLD/Non & & NAFLD/Non & NAFLD/Non & NAFLD/Non & & & \\
\hline & -NAFLD(\%) & -NAFLD(\%) & & -NAFLD(\%) & -NAFLD(\%) & -NAFLD(\%) & & & \\
\hline unmatched & $14.3 \% / 11.4 \%$ & $85.7 \% / 88.6 \%$ & 0.127 & $1.1 \% / 2.1 \%$ & $26.4 \% / 18.7 \%$ & $72.5 \% / 79.2 \%$ & $0.178 / 0.126$ & 0.040 & $98 \%$ \\
\hline matched & $14.3 \% / 9.9 \%$ & $85.7 \% / 90.1 \%$ & 0.045 & $1.4 \% / 1.4 \%$ & $25.8 \% / 17.0 \%$ & $72.8 \% / 81.7 \%$ & $0.428 / 0.503$ & 0.079 & $98 \%$ \\
\hline
\end{tabular}

These data indicate that Sirtuin3 rs28365927 is an independent risk factor for NAFLD that is not associated with age or gender. Individuals carrying an A allele may have significantly increased NAFLD susceptibility in matched populations.

\subsection{Sirtuin3 rs28365927 affects the clinical parameters in NAFLD patients}


To investigate the potential correlation, we compared the Sirtuin3 rs28365927 genotype with the clinical parameters of the NAFLD patients. As shown in Table 5 and Fig. 3, among the NAFLD patients, serum levels of TBIL, DBIL and ALT in rs 28365927 A allele carriers (GA + AA) were 11.1\%, 14.7\% and 41.5\% higher, respectively, than in non-carriers $(G G)(P=0.022, P=0.021, P=0.012$, respectively). In a covariate adjusted linear regression analysis, as shown in Table 6 and Fig. 4, rs28365927 A carriers (AA + AG) were positively correlated with higher ALT among the NAFLD patients $(P=0.045)$.

Table 5

Clinical Characteristics of SIRT3 rs28365927 A Carriers(AA+AG) and Non-Carriers(GG) in the NAFLD Population . $^{\mathrm{a}}$

\begin{tabular}{|c|c|c|c|}
\hline Characteristic & $\begin{array}{l}\text { Carriers } \\
(n=78)\end{array}$ & $\begin{array}{l}\text { Non-Carriers } \\
(n=206)\end{array}$ & P Value \\
\hline Age, y & $49.38 \pm 11.89$ & $48.78 \pm 11.47$ & 0.834 \\
\hline Gender,Male /Female & $48 / 30$ & $123 / 82$ & 0.813 \\
\hline $\mathrm{BMI}, \mathrm{kg} / \mathrm{m}^{2}$ & $25.80 \pm 2.78$ & $26.40 \pm 2.47$ & 0.123 \\
\hline SBP, mmHg & $121.72 \pm 18.15$ & $124.45 \pm 20.35$ & 0.322 \\
\hline DBP, mmHg & $82.28 \pm 14.85$ & $84.73 \pm 15.96$ & 0.623 \\
\hline WC, $\mathrm{cm}$ & $85.02 \pm 8.70$ & $85.64 \pm 8.21$ & 0.747 \\
\hline $\mathrm{HP}, \mathrm{cm}$ & $94.43 \pm 6.29$ & $95.65 \pm 6.88$ & 0.220 \\
\hline $\mathrm{TP}, \mathrm{g} / \mathrm{L}$ & $72.48 \pm 5.33$ & $72.42 \pm 4.81$ & 0.434 \\
\hline Albumin,g/L & $45.00 \pm 4.49$ & $45.00 \pm 4.63$ & 0.529 \\
\hline Globulin,g/L & $27.54 \pm 3.08$ & $27.72 \pm 5.34$ & 0.636 \\
\hline$A / G$ & $1.70 \pm 0.50$ & $1.66 \pm 0.25$ & 0.765 \\
\hline TBIL, umol/L & $13.74 \pm 6.20$ & $12.37 \pm 6.40$ & 0.022 \\
\hline DBIL, umol/L & $6.01 \pm 3.41$ & $5.24 \pm 3.52$ & 0.021 \\
\hline TBA,umol/L & $4.66 \pm 5.13$ & $4.45 \pm 5.00$ & 0.959 \\
\hline $\mathrm{ALT}, \mathrm{U} / \mathrm{L}$ & $38.64 \pm 43.94$ & $27.31 \pm 12.92$ & 0.012 \\
\hline AST, U/L & $30.82 \pm 14.47$ & $28.62 \pm 14.66$ & 0.250 \\
\hline $\mathrm{FBG}, \mathrm{mmol} / \mathrm{L}$ & $5.54 \pm 1.41$ & $5.56 \pm 1.74$ & 0.389 \\
\hline LDL-C, mmol/L & $3.04 \pm 0.80$ & 3.160 .86 & 0.314 \\
\hline $\mathrm{TG}, \mathrm{mmol} / \mathrm{L}$ & $2.17 \pm 0.96$ & $2.66 \pm 2.10$ & 0.318 \\
\hline $\mathrm{TC}, \mathrm{mmol} / \mathrm{L}$ & $5.11 \pm 0.89$ & $5.17 \pm 1.13$ & 0.968 \\
\hline HDL-C, mmol/L & $1.26 \pm 0.30$ & $1.26 \pm 0.33$ & 0.779 \\
\hline \multicolumn{4}{|c|}{$\begin{array}{l}\text { Abbreviations :BMI Body Mass Index,SBP systemic blood pressure,DBP diastolic blood pressure,WC Waist circumference,HP Hip circumference,TP Total } \\
\text { Protein, A/G the ratio of albumin to globulin, TBIL total bilirubin, DBIL direct bilirubin,TBA total bile acid,ALT glutamic-pyruvic transaminase,AST glutamic } \\
\text { oxalacetic transaminase, FBG fasting blood-glucose, LDL-C low density lipoprotein cholesterin, TG triglyceride, TC total cholesterol, HDL-C high density } \\
\text { lipoprotein cholesterol,NAFLD nonalcoholic fatty liver disease. }\end{array}$} \\
\hline
\end{tabular}


Table 6

Significant associations between rs28365927 A Carriers(AA + AG) and Clinical Characteristics in a covariate adjusted linear regression analysis in the NAFLD Population ${ }^{\mathrm{a}}$.

\begin{tabular}{|c|c|c|c|}
\hline Characteristic & $\beta$-coefficient & STAT & P Value \\
\hline $\mathrm{BMI}, \mathrm{kg} / \mathrm{m}^{2}$ & -0.569 & -1.506 & 0.133 \\
\hline SBP, mmHg & -3.082 & -1.117 & 0.265 \\
\hline DBP, mmHg & -2.583 & -1.196 & 0.233 \\
\hline WC, $\mathrm{cm}$ & -0.440 & -0.373 & 0.709 \\
\hline $\mathrm{HP}, \mathrm{cm}$ & -1.148 & -1.196 & 0.233 \\
\hline $\mathrm{TP}, \mathrm{g} / \mathrm{L}$ & 0.116 & 0.185 & 0.853 \\
\hline Albumin,g/L & 0.060 & 0.106 & 0.915 \\
\hline Globulin,g/L & -0.424 & -0.558 & 0.577 \\
\hline$A / G$ & 0.039 & 0.897 & 0.370 \\
\hline TBIL, umol/L & 1.220 & 1.511 & 0.132 \\
\hline DBIL, umol/L & 0.703 & 1.542 & 0.124 \\
\hline TBA,umol/L & 0.154 & 0.156 & 0.876 \\
\hline ALT, U/L & 7.632 & 2.013 & 0.045 \\
\hline AST, U/L & 2.446 & 0.857 & 0.393 \\
\hline $\mathrm{FBG}, \mathrm{mmol} / \mathrm{L}$ & 0.025 & 0.130 & 0.897 \\
\hline LDL-C, mmol/L & -0.101 & -0.903 & 0.367 \\
\hline $\mathrm{TG}, \mathrm{mmol} / \mathrm{L}$ & -0.437 & -1.968 & 0.050 \\
\hline TC, mmol/L & -0.019 & -0.135 & 0.893 \\
\hline $\mathrm{HDL}-\mathrm{C}, \mathrm{mmol} / \mathrm{L}$ & -0.003 & -0.080 & 0.936 \\
\hline \multicolumn{4}{|c|}{$\begin{array}{l}\text { Abbreviations :BMI Body Mass Index,SBP systemic blood pressure,DBP diastolic blood pressure,WC Waist circumference,HP Hip circumference,TP Total } \\
\text { Protein, A/G the ratio of albumin to globulin, TBIL total bilirubin, DBIL direct bilirubin,TBA total bile acid,ALT glutamic-pyruvic transaminase,AST glutamic } \\
\text { oxalacetic transaminase, FBG fasting blood-glucose, LDL-C low density lipoprotein cholesterin, TG triglyceride, TC total cholesterol, HDL-C high density } \\
\text { lipoprotein cholesterol,NAFLD nonalcoholic fatty liver disease. }\end{array}$} \\
\hline
\end{tabular}

\section{Discussion}

NAFLD is a disease that is affected by multifactorial risk [18], among which genetic variation is one of the important factors in individual susceptibility to NAFLD[19]. In this study, we have newly discovered that Sirtuin3 rs28365927 is significantly associated with NAFLD risk, regardless of whether it was in a matched group or not. In the unmatched groups, we found that the Sirtuin3 rs28365927 GA + AA genotype was a significant risk factor for the development of NAFLD in the dominant model, and there was a significant association between Sirtuin3 rs28365927 and higher NAFLD risk in the genotypic model (GA vs GG). In the matched groups, we also found the Sirtuin3 rs28365927 GA + AA genotype was a significant risk factor for the development of NAFLD in the dominant model. There was a significant association between Sirtuin3 rs28365927 and higher NAFLD risk in both the additive and genotypic models (GA vs GG). In contrast, no risk association was discovered for PNPLA3 and other SNP loci, which may be mainly due to different populations. All of the subjects included in this study were of Han Chinese origin, and our conclusions may not be applicable to other ethnicities.

Sirtuins are an evolutionarily conserved NAD ${ }^{+}$-dependent deacetylase family, and there are seven of them in mammals. Three (Sirtuin3, Sirtuin4 and Sirtuin5) are primarily localized in mitochondria[20]. Sirtuin3 is the only sirtuin that has robust deacetylase activity in mitochondria, and it is a crucial gatekeeper of redox status, the epigenetic landscape and lipid homeostasis in hepatocytes[21, 22]. In most cases, increased Sirtuin3 expression is protective from NAFLD. The introduction of Sirtuin3 protects liver function, reduces liver fibrosis, reduces inflammatory response and prevents hepatocyte apoptosis, and the overexpression of Sirtuin3 protects hepatocytes from mitochondrial apoptosis by promoting the mitosis required by Bnip 3[23]. The flavonoid dihydromyricetin can help prevent NAFLD by improving mitochondrial respiration and liver cell redox homeostasis by increasing Sirtuin3 expression[20]. The overexpression of Sirtuin3 in $\mathrm{MTP}^{+/-}$mice significantly reduced the acetylation of MTP as compared with $\beta$-galactosidase controls and increased mitochondrial fatty acid oxidation and reduced hepatic steatosis, CD68 and serum ALT levels[24]. Studies have shown that matrine can inhibit liver steatosis and liver inflammation in NAFLD by promoting the metabolism of acylcarnitine through Sirtuin3/LCAD signalling pathways[25]. In mice, Berberine reduced the NAFLD induced by a high-fat diet by activating Sirtuin3[26]. Protocatechuic acid has a protective effect on NAFLD by increasing Sirtuin3 expression[27]. Interestingly, Sirtuin3 overexpression makes the liver and hepatocytes susceptible to palmitate-induced cell death[28]. So far, no studies have reported the relationship between Sirtuin3 genetic variation and NAFLD. In this study, among the NAFLD patients, we found that serum levels of TBIL, DBIL and ALT in rs28365927 A allele 
carriers (GA + AA) were $11.1 \%, 14.7 \%$ and $41.5 \%$ higher, respectively, than in non-carriers (GG) (Fig. 3). In addition, among the NAFLD patients, Rs28365927 A carriers $(A A+A G)$ were positively correlated with higher ALT (Fig. 4). These results indicate that the Sirtuin3 rs28365927 A allele may be associated with an impairment of liver function.

ALT level is one of the high risk indexes for NAFLD, and Sirtuin3 rs28365927 may contribute to the risk for NAFLD through an effect on ALT level. NAFLD has been strongly associated with ALT activity in previous studies[29-31]. Mild ALT elevation is largely attributed to NAFLD[32, 33]. Sirtuin3 rs28365927 leads to the production of reactive oxygen species (ROS) by decreasing the activity of mitochondrial respiratory chain (MRC), inactivating the extracellular signal regulated kinase-cAMP response element binding protein (ERK-CREB) pathway and reducing the activity of mitochondrial trifunctional protein (MTP)/ long chain acyl coa dehydrogenase (LCAD)/superoxide dismutase 2 (SOD2), and the production of ROS leads to the death of liver cells, which in turn leads to an increase in serum ALT levels (Fig. 5) [2, 23-26, 34-37]. However, the potential mechanism of Sirtuin3 rs28365927 in increasing NAFLD susceptibility requires further study.

In conclusion, this study is the first comprehensive case-controlled study to research the relationship between genetic variants of the Sirtuin3 gene and susceptibility to NAFLD, and these findings are important because they provide a new point for exploring the role that Sirtuin3 plays in NAFLD based on genetic factors. Sirtuin3 rs28365927 is a newly discovered independent risk factor SNP associated with NAFLD in the Han Chinese population. The Rs28365927 A allele significantly increased the ALT levels of NAFLD patients, and more studies are needed to clarify the underlying mechanism.

\section{Abbreviations}

PGC1 $\beta$, peroxisome proliferator-activated receptor- $\gamma$ coactivator-1 $\beta$; FASN, fatty acid synthase; mTOR, mechanistic target of rapamycin; PEMT, phosphatidylethanolamine N-methyltransferase; MTTP, microsomal triglyceride transfer protein; SLC27A5, soluble carrier family 27 member A5; APOE, Apolipoprotein E; CYP2E1, cytochrome P450 2E1; PPARG, Peroxisome proliferator-activated receptor-y; TCF7L2, transcription factor 7-like 2; HIF3A, Hypoxia Inducible Factor 3 Alpha Subunit; PNPLA3, patatin-like phospholipase domain containing 3; FAS, fas cell surface death receptor; SIRT3, Sirtuin3; IL-6, interleukin-6; STAT2, signal transducer and activator of transcription 2; TLR4, toll like receptor 4; BMI Body Mass Index,SBP systemic blood pressure,DBP diastolic blood pressure,WC Waist circumference,HP Hip circumference,TP Total Protein, A/G the ratio of albumin to globulin, TBIL total bilirubin, DBIL direct bilirubin,TBA total bile acid,ALT glutamic-pyruvic transaminase,AST glutamic oxalacetic transaminase, FBG fasting blood-glucose, LDL-C low density lipoprotein cholesterin, TG triglyceride, TC total cholesterol, HDL-C high density lipoprotein cholesterol,NAFLD nonalcoholic fatty liver disease;

\section{Declarations}

Acknowledgments

We would like to thank the Health Management Center of Xiangya Hospital for the recruitment of subjects.

Funding

This work was supported by the National Scientific Foundation of China (grant 81974513, 81302850), the National Science and Technology Plan of China (grant 2017ZX09304014). The funders had no role in the study design, data collection and analysis, decision to publish, or preparation of the manuscript.

Availability of data and materials

None.

Authors' contributions

All the authors contributed to this work by recruiting the subjects or performing the genetic and statistical analysis.

Ethics approval and consent to participate

This case-control retrospective study was approved by the Medical Ethics Committee of the Department of Clinical Pharmacology, Xiangya Hospital, Central South University (Changsha, China) and was registered with the Chinese Clinical Trial Registry (no. ChiCTR-ROC-15006899). All subjects gave written and informed consent before participating in this study.

Consent for publication

Not applicable.

Competing interests

The authors have declared that no competing interests exist.

\section{References}

1. Rinella M, Charlton M. The globalization of nonalcoholic fatty liver disease: Prevalence and impact on world health. Hepatology. 2016;64(1):19-22.

2. Brunt EM, et al. Nonalcoholic fatty liver disease. Nat Rev Dis Primers. 2015;1:15080.

3. Reimer KC, et al. New drugs for NAFLD: lessons from basic models to the clinic. Hepatol Int. 2020;14(1):8-23.

Page 9/14 
4. Brea A, et al. Nonalcoholic fatty liver disease is associated with carotid atherosclerosis: a case-control study. Arterioscler Thromb Vasc Biol. 2005;25(5):1045-50.

5. Stahl EC, et al. Macrophages in the Aging Liver and Age-Related Liver Disease. Front Immunol. 2018;9:2795.

6. Kitade H, et al., Nonalcoholic Fatty Liver Disease and Insulin Resistance: New Insights and Potential New Treatments. Nutrients, 2017. 9(4).

7. Tilg H, Moschen AR, Roden M. NAFLD and diabetes mellitus. Nat Rev Gastroenterol Hepatol. 2017;14(1):32-42.

8. Mota M, et al. Molecular mechanisms of lipotoxicity and glucotoxicity in nonalcoholic fatty liver disease. Metabolism. 2016;65(8):1049-61.

9. Fan JG, Kim SU, Wong VW. New trends on obesity and NAFLD in Asia. J Hepatol. 2017;67(4):862-73.

10. Katsiki N, Mikhailidis DP, Mantzoros CS. Non-alcoholic fatty liver disease and dyslipidemia: An update. Metabolism. 2016;65(8):1109-23.

11. Del Campo JA, et al., Genetic and Epigenetic Regulation in Nonalcoholic Fatty Liver Disease (NAFLD). Int J Mol Sci, 2018. 19(3).

12. Valenti L, et al. Homozygosity for the patatin-like phospholipase-3/adiponutrin I148M polymorphism influences liver fibrosis in patients with nonalcoholic fatty liver disease. Hepatology. 2010;51(4):1209-17.

13. Vespasiani-Gentilucci U, et al. Promoting genetics in non-alcoholic fatty liver disease: Combined risk score through polymorphisms and clinical variables. World J Gastroenterol. 2018;24(43):4835-45.

14. Wang Y, et al. Relationship of SREBP-2 rs2228314 G > C polymorphism with nonalcoholic fatty liver disease in a Han Chinese population. Genet Test Mol Biomarkers. 2014;18(9):653-7.

15. Eslam M, Valenti L, Romeo S. Genetics and epigenetics of NAFLD and NASH: Clinical impact. J Hepatol. 2018;68(2):268-79.

16. F JG. Guidelines for the diagnosis and treatment of nonalcoholic fatty liver disease. Chin J Clini Hepatol, 2010. 26.

17. Shi YY, He L. SHEsis, a powerful software platform for analyses of linkage disequilibrium, haplotype construction, and genetic association at polymorphism loci. Cell Res. 2005;15(2):97-8.

18. Younossi Z, et al. Global burden of NAFLD and NASH: trends, predictions, risk factors and prevention. Nat Rev Gastroenterol Hepatol. 2018;15(1):11-20.

19. Sookoian S, Pirola CJ. Genetic predisposition in nonalcoholic fatty liver disease. Clin Mol Hepatol. 2017;23(1):1-12.

20. Zeng X, et al. Dihydromyricetin Ameliorates Nonalcoholic Fatty Liver Disease by Improving Mitochondrial Respiratory Capacity and Redox Homeostasis Through Modulation of SIRT3 Signaling. Antioxid Redox Signal. 2019;30(2):163-83.

21. Ahn BH, et al. A role for the mitochondrial deacetylase Sirt3 in regulating energy homeostasis. Proc Natl Acad Sci U S A. 2008;105(38):14447-52.

22. Podrini C, et al. Redox homeostasis and epigenetics in non-alcoholic fatty liver disease (NAFLD). Curr Pharm Des. 2013;19(15):2737-46.

23. Li R, et al. Therapeutic effect of Sirtuin 3 on ameliorating nonalcoholic fatty liver disease: The role of the ERK-CREB pathway and Bnip3-mediated mitophagy. Redox Biol. 2018;18:229-43.

24. Nassir F, et al. Regulation of mitochondrial trifunctional protein modulates nonalcoholic fatty liver disease in mice. J Lipid Res. 2018;59(6):967-73. 25. Wang GE, et al. Theacrine protects against nonalcoholic fatty liver disease by regulating acylcarnitine metabolism. Metabolism. 2018;85:227-39.

26. Xu X, et al. Berberine alleviates nonalcoholic fatty liver induced by a high-fat diet in mice by activating SIRT3. FASEB J. 2019;33(6):7289-300.

27. Sun R, et al. Sirtuin 3-mediated deacetylation of acyl-CoA synthetase family member 3 by protocatechuic acid attenuates non-alcoholic fatty liver disease. Br J Pharmacol. 2020;177(18):4166-80.

28. Li S, et al. Sirtuin 3 acts as a negative regulator of autophagy dictating hepatocyte susceptibility to lipotoxicity. Hepatology. 2017;66(3):936-52.

29. Clark JM, Brancati FL, Diehl AM. The prevalence and etiology of elevated aminotransferase levels in the United States. Am J Gastroenterol. 2003;98(5):960-7.

30. Chen $\mathrm{CH}$, et al. Prevalence and etiology of elevated serum alanine aminotransferase level in an adult population in Taiwan. J Gastroenterol Hepatol. 2007;22(9):1482-9.

31. Pendino GM, et al. Prevalence and etiology of altered liver tests: a population-based survey in a Mediterranean town. Hepatology. 2005;41(5):1151-9.

32. Giannini EG, Testa R, Savarino V. Liver enzyme alteration: a guide for clinicians. CMAJ. 2005;172(3):367-79.

33. Liangpunsakul S, Chalasani N. Unexplained elevations in alanine aminotransferase in individuals with the metabolic syndrome: results from the third National Health and Nutrition Survey (NHANES III). Am J Med Sci. 2005;329(3):111-6.

34. Qiu X, et al. Calorie restriction reduces oxidative stress by SIRT3-mediated SOD2 activation. Cell Metab. 2010;12(6):662-7.

35. Tao R, et al. Sirt3-mediated deacetylation of evolutionarily conserved lysine 122 regulates MnSOD activity in response to stress. Mol Cell. 2010;40(6):893-904.

36. Begriche K, et al. Mitochondrial adaptations and dysfunctions in nonalcoholic fatty liver disease. Hepatology. 2013;58(4):1497-507.

37. Luedde T, Kaplowitz N, Schwabe RF. Cell death and cell death responses in liver disease: mechanisms and clinical relevance. Gastroenterology, 2014. 147(4): 765-83 e4.

\section{Figures}




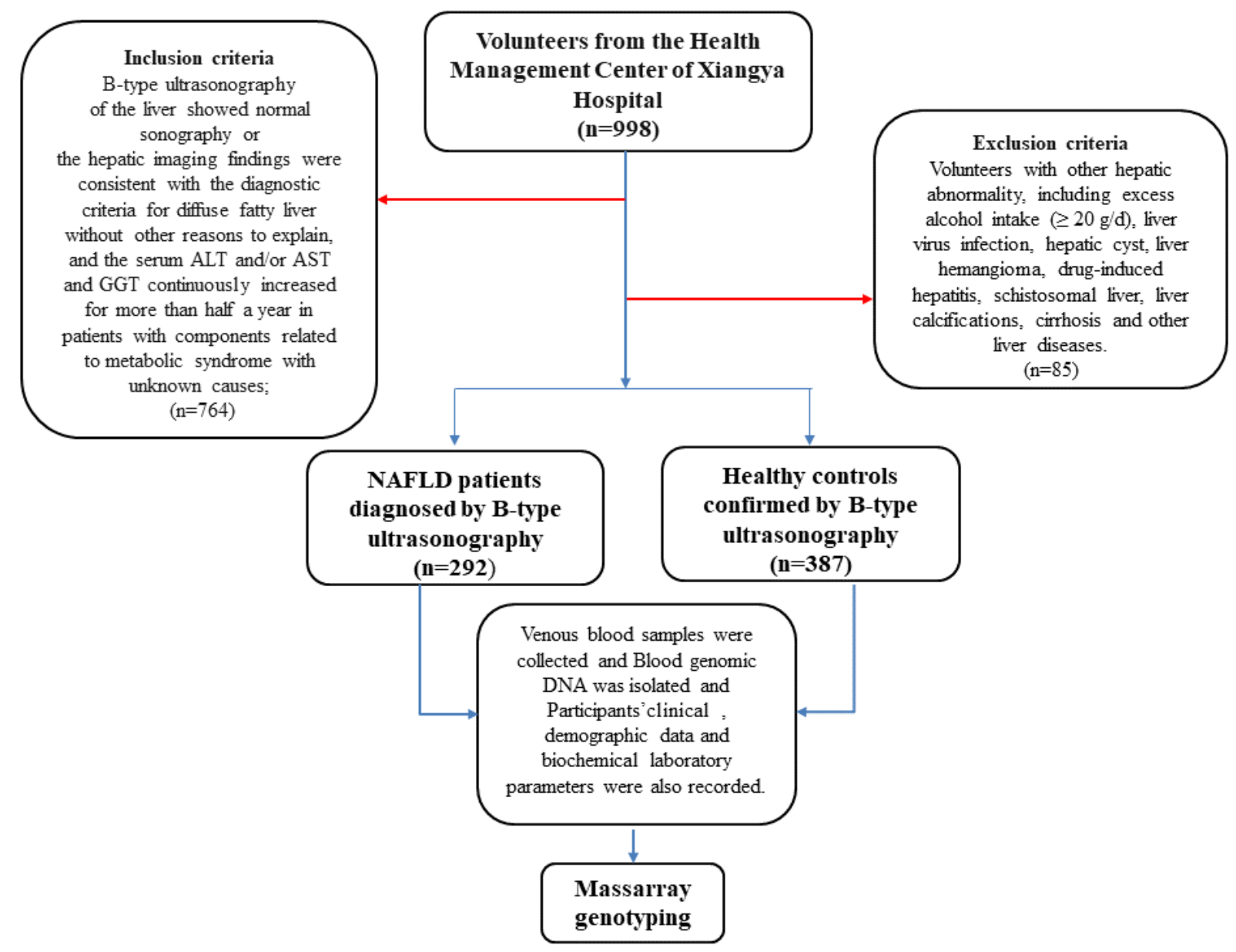

Figure 1

Diagram of the screening procedure for patients of NAFLD and non-NAFLD. Abbreviations: NAFLD, non-alcoholic fatty liver disease; ALT, glutamic-pyruvic transaminase; AST, glutamic oxalacetic transaminase; GGT, gamma-glutamyl transferase; 


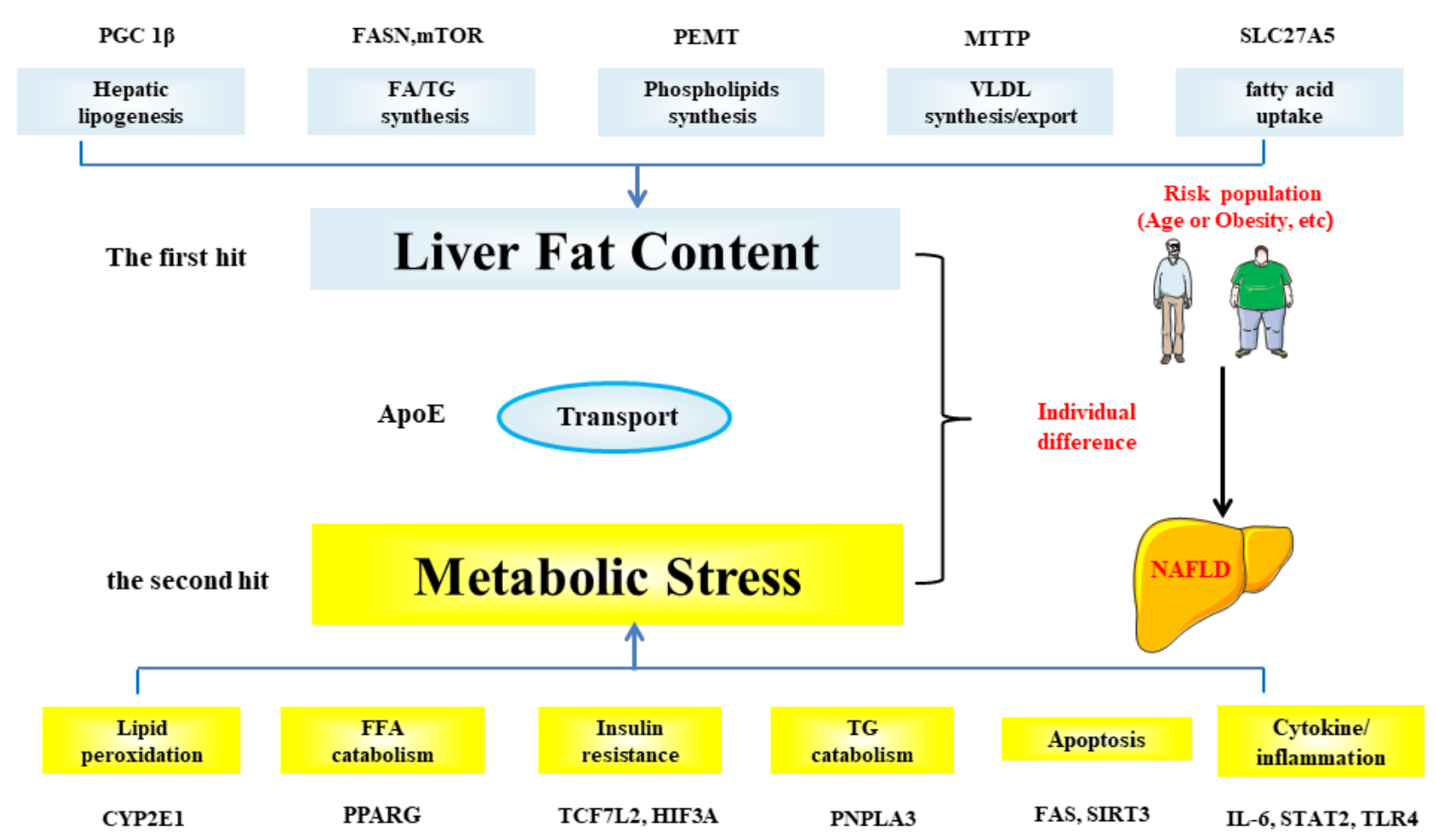

Figure 2

Individual susceptibility genes were selected based on the idea of "two-hit theory" affecting individual susceptibility risk of NAFLD. Among them, 7 genes (MTTP, PEMT, FASN, PGC1ß, ApoE, mTOR and SLC27A5) were related to "first hit". 10 genes (PNPLA3, Sirtuin3, CYP2E1, Fas, TLR4, TCF7L2, PPARG, IL6, STAT2 and HIF3A) were associated with "second hit ". Abbreviations: PGC1 $\beta$, peroxisome proliferator-activated receptor- $\gamma$ coactivator-1 $\beta$; FASN, fatty acid synthase; mTOR, mechanistic target of rapamycin;PEMT, phosphatidylethanolamine N-methyltransferase; MTTP, microsomal triglyceride transfer protein; SLC27A5, soluble carrier family 27 member A5;APOE, Apolipoprotein E; CYP2E1, cytochrome P450 2E1; PPARG, Peroxisome proliferator-activated receptor-ץ; TCF7L2, transcription factor 7-like 2; HIF3A, Hypoxia Inducible Factor 3 Alpha Subunit; PNPLA3, patatin-like phospholipase domain containing 3; FAS, fas cell surface death receptor; SIRT3, Sirtuin3; IL-6, interleukin-6; STAT2, signal transducer and activator of transcription 2; TLR4, toll like receptor 4;

total bilirubin

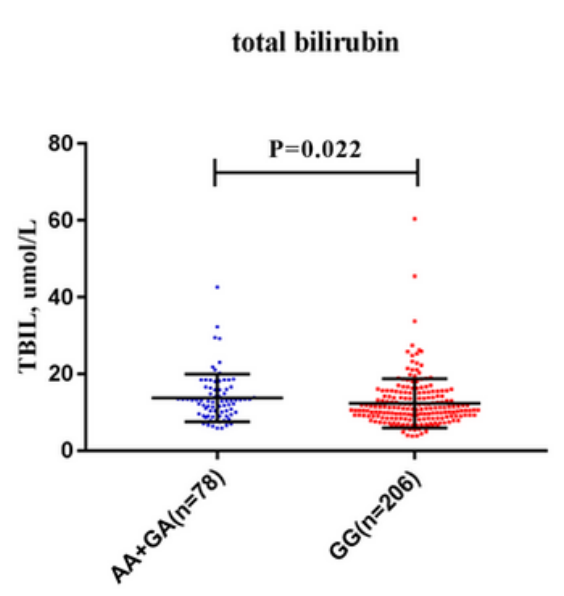

A

SIRT3 rs28365927 genotype

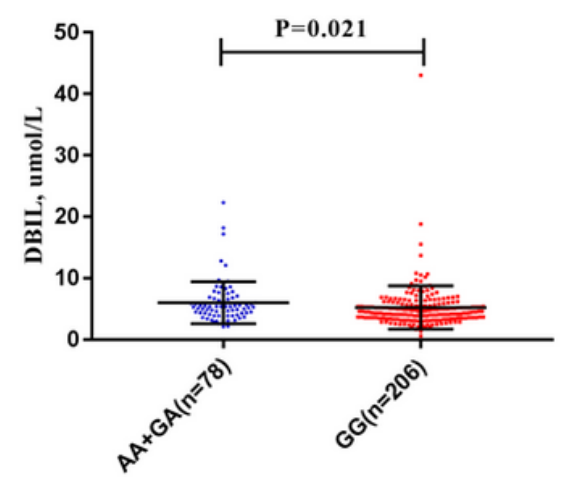

B SIRT3 rs28365927 genotype glutamic-pyruvic transaminase

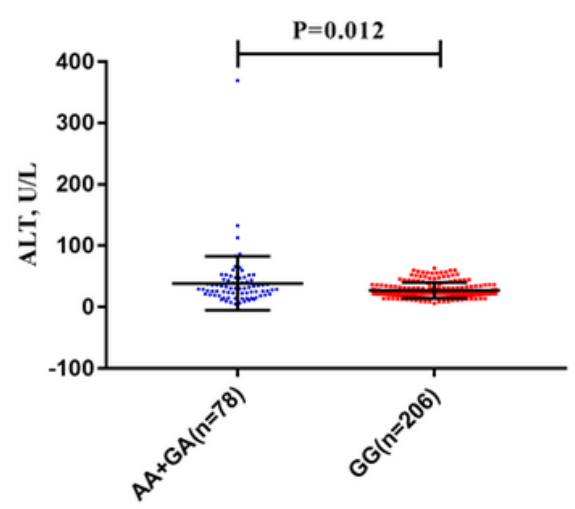

C

SIRT3 rs28365927 genotype

Figure 3

Comparison of Clinical Characteristics of SIRT3 rs28365927 genotype in NAFLD patients. A, Comparison of TBIL values between the SIRT3 rs28365927 AA/GA and GG genotype groups. B, Comparison of DBIL values between the SIRT3 rs28365927 AA/GA and GG genotype groups. C, Comparison of ALT values 
between the SIRT3 rs28365927 AA/GA and GG genotype groups. Values are expressed as mean \pm SD and compared by Student's t-test if the data is normally distributed, otherwise Mann-Whitney U test is used, except for gender that $\mathrm{p}$ value stands for statistical significance using Chi-square test. P-value $\mathbb{0} 0.05$ considered as statistically significant (in bold). Abbreviations: NAFLD, non-alcoholic fatty liver disease邓SIRT3, Sirtuin3; TBIL, total bilirubin; DBIL, direct bilirubin; ALT, glutamic-pyruvic transaminase;

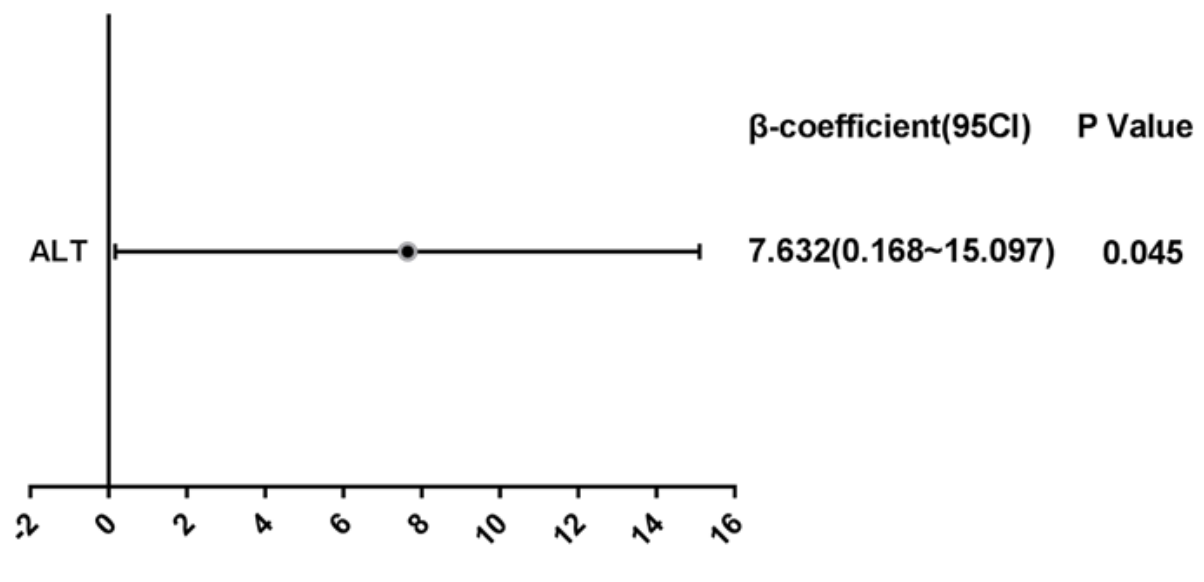

$\beta$-coefficient(95\%Cl)

\section{Figure 4}

Significant associations between rs28365927 A Carriers(AA+AG) and ALT in NAFLD patients in a covariate adjusted linear regression analysis. $\beta$-coefficient: estimated quantitative effect of rs28365927 A Carriers on a phenotype of NAFLD, P: level of statistical significance of the covariate(age and gender) adjusted linear regression analysis. P-value区0.05 considered as statistically significant (in bold). Abbreviations: NAFLD, non-alcoholic fatty liver disease邓SIRT3, Sirtuin3; ALT, glutamic-pyruvic transaminase; 


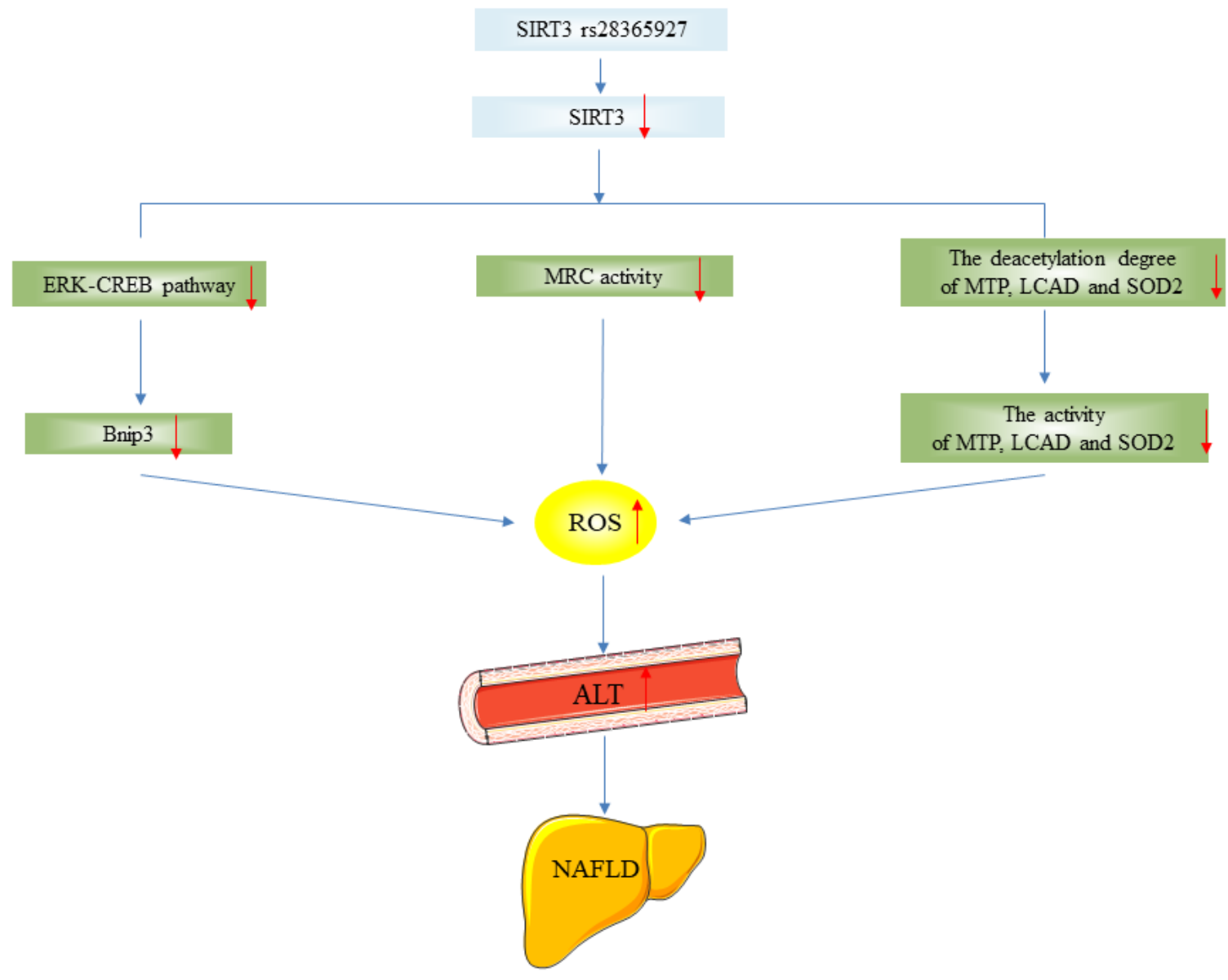

Figure 5

The potential mechanism pathway of SIRT3 rs28365927 to increase the risk of NAFLD. Sirtuin3 rs28365927 leads to the production of reactive oxygen species(ROS) by decreasing the activity of MRC, inactivating the ERK-CREB pathway and reducing the activity of MTP/LCAD/SOD2, and the production of ROS leads to the death of liver cells, which in turn leads to an increase in serum ALT levels.[2, 23-26, 34-37]. Abbreviations: SIRT3, Sirtuin3; MRC,mitochondrial respiratory chain; ROS, reactive oxygen species; ERK,extracellular signal regulated kinase; CREB,the cAMP response element binding protein; Bnip3,BCL2 interacting protein 3; MTP,mitochondrial trifunctional protein; LCAD,long chain acyl coa dehydrogenase; SOD2,superoxide dismutase 2; NAFLD,Non-alcoholic fatty liver disease. 